\title{
Estado, democracia y fuerza pública ${ }^{1}$
}

\section{State, democracy and Army}

\author{
YADIRA ESTHER GARCÍA GARCÍA \\ Especialista en Estudios politicos-Económicos y Magíster en Desarrollo Social. Docente investigadora del grupo de \\ investigación en Derecho, Política y Sociedad, de la Facultad de Derecho \\ yadira2705@yahoo.es
}

Recibido: 20 de Septiembre de 2012

Aceptado: 15 de Noviembre de 2012

\section{RESUMEN}

Dispone el artículo 216 de la Constitución Política de Colombia "Todos los colombianos están obligados a tomar las armas cuando las necesidades públicas lo exijan para defender la independencia nacional y las instituciones públicas". Recordar el desplazamiento es tener presente la ola de terror vivida en toda Colombia y en especial en la zona de los Montes de María. La investigación desarrollada se propuso narrar los hechos violentos ocurridos en el municipio de San Jacinto Bolívar entre los años 1997 al 2002, este artículo de reflexión se trata de destacar la soberanía del Estado y el papel de la fuerza pública en su lucha por mantener el control y el orden territorial dentro de la región, en colaboración con la comunidad.

Palabras clave: Desplazamiento, Terror, Fuerza Pública y Control territorial.

\begin{abstract}
The 216 article of the Colombian Constitution states that all Colombians are forced to take up arms to defend national independence and public institutions. The displacement of people in the area of Montes de María reminds the wave of terror across Colombia. This research narrates the violent events in the municipality of San Jacinto Bolivar from 1997 to 2002. Also, it attempts to highlight reflection of state sovereignty and the role of the security forces in their fight for control and territorial order in the region, in collaboration with the community.

Key words: Displacement, Terror, forces and territorial control.
\end{abstract}

\section{Introducción}

San Jacinto Bolívar (Colombia) es un municipio ubicado en los Montes de María, con una población actual de 21.218 habitantes. En los últimos años la población ha presentado una tendencia de concentración de sus habitantes hacia la cabecera municipal, como consecuencia de los fuertes combates registrados en la región. El flagelo de la guerra (que desde hace décadas vive Colombia) azotò muy fuertemente la zona de los Montes de María y en especial al departamento de Bolívar en los años 1997 al 2002, momento en el cual se presentaron masacres, intimidaciones y violación de derechos humanos, los cuales terminaron en un masivo desplazamiento de los habitantes rurales hacia la zona urbana.

1 El presente artículo es producto de la investigación titulada "fuerza pública frente a la población desplazada en la recuperación de la gobernabilidad en seguridad democrática", desarrollada dentro del grupo de investigación en derecho, política y sociedad. de la Universidad de la Costa. Categoría B de CoLciencias. Barranquilla, Colombia. 
Actualmente el municipio de San Jacinto, Bolívar está dividido en 42 barrios, en la zona urbana, y en la parte rural encontramos 7 corregimientos y 13 veredas. Los desplazamientos en masas obligaron a estas familias a ubicarse en las afueras de la población invadiendo terrenos cercanos, que con el pasar de los años lograron que la administración pública les adjudicara los lotes. Hoy en día estos barrios producto del desplazamiento toman los nombres como son: la Paz, la Campesina, Anzuelo y Ocho de diciembre, Javier cirujano, entre otros.

La violencia es una forma de acción humana a través de la cual se agrede a otras personas causándoles daño, afectando su integridad, sus sentimientos, su dignidad e incluso su vida, en la búsqueda de algún fin "superior", de acuerdo con los criterios valorativos, creencias y normas del actor que la ejerce (Villa Gómez, 2007:17).

Entre los años 1996 al 2002 San Jacinto Bolívar, vivió momentos difíciles por cuenta del orden público, el accionar de los grupos armados ilegales, dejando muertos, desaparecidos, y por ende desplazamientos masivos e individuales, propiciando desolación y hogares dispersos.

Con la consolidación de la política de la seguridad democrática, la población más vulnerable empieza a confiar en la efectividad de la fuerza pública, sin embargo antes de que se diera esta consolidación no había una capacidad efectiva de respuesta de la fuerza pública para atender y cubrir todas las necesidades en cuanto a seguridad requerida por la sociedad para defender la soberanía y los derechos de esta población.

\section{Metodología}

La investigación presentada está enfocada desde un punto de vista cuantitativo, con un enfoque narrativodescriptivo de los hechos. Es narrativo porque se puede observar las transformaciones y los grandes avances logrados por la fuerza pública en la recuperación, consolidación y control territorial de las zonas desplazadas las cuales estaban siendo controladas por los grupos terrorista. La muestra para desarrollar esta investigación se tomó de manera intencional en 4 corregimientos ubicados en la zona rural del municipio como lo son: Arenas, Bajo Grande, Las Charquitas y Las Palmas. Los corregimientos mencionados anteriormente se ubicaron después del desplazamiento en la zona urbana rural del municipio de
San Jacinto Bolívar, dando origen a nuevos barrios como: Ocho de Diciembre, El Anzuelo, La Paz, La Campesina, Ocho de diciembre y Javier Cirujano, entre otros.

Los instrumentos que se aplicaron fueron las entrevistas, las fotografías, las cuales evidencia la situación actual de la comunidad.

\section{Marco Teórico}

\section{Recuerdos del desplazamiento}

El conflicto armado vivido en los años 1997 a 2002 generó grandes niveles de desplazamiento forzado viéndose obligados a abandonar sus caseríos y asentarse en los cascos urbanos provocando así un desajuste en la vida social, familiar y económica en todos sus afectados como consecuencias de las muertes de algunos familiares, incluyendo torturas, amenazas y otras atrocidades.

En los Montes de María la aparición de la AUC, está ligada aproximadamente desde el año 1990 con muy poca participación, hasta que en el año 1996 cuando las clases elite, junto con Salvatore Mancuso deciden proteger sus tierras de los frentes 35 - 37 de las FARC que tenían a esta población azotada con sus secuestros, extorsiones y crímenes indiscriminados. A partir de esta fecha 1996 se registra la primera masacre por las autodefensas la cual tuvo lugar en el Guamo Bolívar con el asesinato de 4 personas. Desde este momento empieza una lucha constante a muerte por el dominio de estas tierras FARC vs. AUC. (GARCIA, Y. Tesis, 2011:43-44).

En el año 1996, San Jacinto Empieza a vivir por primera vez, la ola de terror, miedo y zozobra de los grupos al margen de la ley, pero sólo para el año 1997, es cuando realmente el caos y las masacres se apoderan de estas zonas rurales.

La tabla anterior indica que los desplazamientos ocurridos en los cinco corregimientos del municipio de San Jacinto, Bolívar en el año de 1997, obedecen a factores únicos como son los enfrentamientos entre los grupos armados de las AUC y las FARC, que al final desencadenaron masacres entre familias inocentes de este conflicto. Las familias desplazadas sumaron 472, para un total de 1669 personas, que huyeron de sus tierras hacia el municipio. 
Tabla 1. Desplazamientos de los corregimientos más afectados.

\begin{tabular}{|c|l|l|c|c|}
\hline \multicolumn{2}{|c|}{ DESPLAZAMIENTOS } & \multicolumn{1}{|c|}{ MOTIVO } & N $^{\text {o }}$ FAMILIAS & POBLACIÓN \\
\hline FECHA & \multicolumn{1}{|c|}{ POBLACIÓN } & \multicolumn{1}{|c|}{176} \\
\hline $12-02-97$ & Las Charquitas & $\begin{array}{l}\text { Enfrentamientos grupos armados no } \\
\text { identificados }\end{array}$ & 49 & 363 \\
\hline $13-03-99$ & Arenas & Masacre realizada por Autodefensas & 98 & 503 \\
\hline $22-10-99$ & Bajo Grande & Masacre realizada por Autodefensas & 128 & 627 \\
\hline
\end{tabular}

Fuente: Oficina de acción social de la Alcaldía de San Jacinto Bolívar. 2008.

Población desplazada por rango de edades. Año 1997

Tabla 2

\begin{tabular}{|c|c|c|}
\hline \multicolumn{3}{|c|}{$\begin{array}{c}\text { Población por rango de edades. } \\
\text { Corregimiento Las Palmas }\end{array}$} \\
\hline Nivel & $\mathbf{N}^{\mathbf{0}}$ de personas & $\%$ \\
\hline $6-5$ & 69 & 6.43 \\
\hline $11-14$ & 110 & 10.25 \\
\hline $15-19$ & 115 & 10.71 \\
\hline $20-24$ & 136 & 12.67 \\
\hline $25-29$ & 112 & 10.43 \\
\hline Mayores de 30 & 102 & 9.50 \\
\hline Mayores de 60 & 195 & 18.17 \\
\hline Total & 234 & 21.80 \\
\hline
\end{tabular}

Tabla 4

\begin{tabular}{|c|c|c|}
\hline \multicolumn{3}{|c|}{$\begin{array}{c}\text { Población por rango de edades. } \\
\text { Corregimiento Arenas }\end{array}$} \\
\hline Nivel & N $^{\text {o }}$ de personas & $\%$ \\
\hline $0-5$ & 74 & 13.02 \\
\hline $6-10$ & 82 & 14.43 \\
\hline $11-14$ & 57 & 10.03 \\
\hline $15-19$ & 69 & 12.14 \\
\hline $20-24$ & 51 & 8.97 \\
\hline $25-29$ & 36 & 6.33 \\
\hline Mayores de 30 & 162 & 28.52 \\
\hline Mayores de 60 & 37 & 6.51 \\
\hline Total & 568 & $100 \%$ \\
\hline
\end{tabular}

Tabla 3

\begin{tabular}{|c|c|c|}
\hline \multicolumn{3}{|c|}{$\begin{array}{c}\text { Población por rango de edades. } \\
\text { Corregimiento Bajo Grande }\end{array}$} \\
\hline Nivel & N $^{\text {o }}$ de personas & $\%$ \\
\hline $6-5$ & 11 & 2.88 \\
\hline $11-14$ & 53 & 13.91 \\
\hline $15-19$ & 26 & 6.82 \\
\hline $20-24$ & 41 & 10.76 \\
\hline $25-29$ & 31 & 5.51 \\
\hline Mayores de 30 & 35 & 9.18 \\
\hline Mayores de 60 & 123 & 32.28 \\
\hline Total & 61 & 16.01 \\
\hline
\end{tabular}

Tabla 5

\begin{tabular}{|c|c|c|}
\hline \multicolumn{3}{|c|}{$\begin{array}{c}\text { Población por rango de edades. } \\
\text { Corregimiento Las Charquitas }\end{array}$} \\
\hline Nivel & N $^{\mathbf{0}}$ de personas & $\%$ \\
\hline $0-5$ & 14 & 9.52 \\
\hline $6-10$ & 19 & 12.92 \\
\hline $11-14$ & 16 & 10.88 \\
\hline $15-19$ & 22 & 14.96 \\
\hline $20-24$ & 16 & 10.88 \\
\hline $25-29$ & 9 & 6.12 \\
\hline Mayores de 30 & 35 & 23.84 \\
\hline Mayores de 60 & 16 & 10.88 \\
\hline Total & 147 & $100 \%$ \\
\hline
\end{tabular}


Las tablas anteriores corresponden a la población desplazada, por rango de edades en los cuatro corregimientos de la zona rural de San Jacinto, Bolívar, la tabla $\mathrm{N}^{\circ} 2$, muestra que los niños entre los 0-10 años fueron 179 y los adultos mayores 234 del corregimiento de las Palmas fueron los más afectados, quienes por su condición de desplazados se vieron muy afectados en cuanto a su parte física y psicosocial. Este desplazamiento en gran escala genero unas fuertes medidas de orden público, por parte de la administración pública quien estuvo presente en la reubicación de estas familias.

\section{Consolidación de la democracia actual}

Si se compara la seguridad de los colombianos en los años 60 hasta los 90, se encuentra un periodo de violencia, enmarcado por el secuestro, los asesinatos y los atentados por parte de grupos guerrilleros y los grupos narcotraficantes, quiénes se disputan parte de los territorios de la geografía colombiana, en este preciso momento el pueblo pierde la poca fe y su convencimiento hacia el Estado, ya que este no presentaba unas políticas fuertes que garantizaran los derechos a la población. Esta debilidad evidencio el mal ordenamiento del estado y sus fuerzas públicas; el descontento de la ciudadanía, los homicidios por parte de los grupos narcotraficantes contra la sociedad civil, los secuestros y atentados mostraban esta gran problemática.

Como consecuencia es otro error de la política de Aristóteles que en un gobierno bien ordenado no deben gobernar los hombres, sino las leyes. ¡Qué hombre que este en su sano juicio aunque no saben leer, ni escribir, viéndose gobernado por aquel a quien teme no creerá que este pueda matarle y haciéndole daño si lo le obedece? (Tierno Galvan, E. 2007:206).

El Estado como garante de nuestro derechos está en la obligación a través de sus leyes de salvaguardarnos en caso excepcionales por lo cual este "es la sociedad política y jurídicamente organizada, con la autoridad suficiente para imponer un orden jurídico dentro de su propio territorio y para hacer respetar su personalidad ante el concierto internacional" (Younes Moreno, D. 2009:273).

La política de los años 2002 al 2010, mostró una nueva forma de seguridad para los colombianos, su dirigente el presidente de la Republica el Dr. Álvaro Uribe Vélez, implemento unos lineamientos con unos objetivos específicos para terminar con el flagelo del terror, el cual se había apoderado de muchas zonas estratégicas de la geografía Colombia, entre ellas la zona de los Montes de María. Estos lineamientos estaban encaminados a garantizar la libertad y la seguridad democrática en todo el país.
La libertad de la cual se hace mención tan frecuente y honrosa en las historias y en la filosofía de los antiguos griegos y romanos y en los escritos y discursos de quienes de ellos han recibido toda su educación en materia de política, no es libertad de los hombres particulares, sino la libertad del estado que coincide con que cada hombre tendría si no existieran leyes civiles ni estado, en absoluto (Tierno Galvan, E.2007:163-164).

El gobierno colombiano empieza su proceso de libertad y seguridad a partir del año 2002, el cual se emprende una etapa de asentamiento de las fuerzas militares y la policía nacional, basándose en una política de seguridad democrática, considerada como la mayor ofensiva que jamás se haya realizado contra organizaciones al margen de la ley y los grupos paramilitares. Sin embargo fue tan solo a comienzos año 2007 en donde se hacen fuertes ajustes y reflexiones para consolidar la política de defensa y seguridad democrática.

...La Política de Defensa y Seguridad Democrática (PDSD) desarrollada en el cuatrienio 2002-2006 probó ser una estrategia exitosa en la lucha contra el terrorismo y el narcotráfico, así como una herramienta fundamental en la recuperación del control territorial. Después de cuatro años de implementación, los resultados son evidentes. Hoy es indiscutible el hecho de que todos los indicadores de violencia y criminalidad se han reducido de manera sustancial, generando un clima de confianza y optimismo, Esta situación impone una serie de retos institucionales para el gobierno de Colombia y su Fuerza Pública. (CONPES 3460, 2007:1).

El Concejo Nacional de Política Económica y Social CONPES 3460, dentro de sus principales objetivos se encuentra mantener el control y la gobernabilidad total del territorio nacional, y para ello ha aumentado el número de integrantes de la fuerza pública y los ha desplegado sobre casi todo el territorio nacional, para conseguir un control y mejor protección para la sociedad civil. El estado al adoptar esta estrategia logró ganar la confianza de la población, a través de la protección de su seguridad.

\section{Consolidación de la democracia en San Jacinto Bolívar}

En la región de los Montes de María la población y la fuerza pública han sido el pilar fundamental para la construcción de este documento, los cuales dan fe del ambiente de tranquilidad del que goza actualmente la población, reconociendo que en estos momentos, existen aún pequeños brotes de delincuencia común. 
El Subcomisario de policía de San Jacinto, Bolívar, Stanley Barrios Valencia, sostiene que: "la seguridad en el municipio de San Jacinto se encuentra controlada tanto en la cabecera municipal como en el área rural, y para ello se han implementado medidas como el patrullaje, las requisas e identificación de personas, los allanamientos, el puerta a puerta (que consiste en un censo en conjunto con la comunidad donde entregan información sobre problemáticas y se produce una reacción inmediata por parte de la policía), además se efectúan periódicamente reuniones con los líderes de las comunidades en cada uno de los barrios donde existe inseguridad.

Los jóvenes también han sido constructores de esta seguridad, con ellos se han creado grupos interdisciplinarios en los sectores más afectados, debido al gran número de adolescentes rebeldes. El trabajo en la comunidad ha sido mancomunado, se ha venido trabajando arduamente para combatir la drogadicción, la prostitución y la violencia intrafamiliar. El índice de capturas ha disminuido hoy existe un promedio de cuatro capturas al mes de expendedores de drogas los cuales se colocan a disposición de la fiscalía para que ella tome las medidas pertinentes.

Actualmente la población de los Montes de María goza de una paz pues se han erradicado los grupos al margen de la ley, no se presentan denuncias de desparecidos, ni casos de desplazamiento forzoso, las personas incluso están retornando a sus lugares de origen, hay una confianza y credibilidad en la fuerza pública lo que hace que la comunidad colabore activamente en la consolidación de la seguridad.

La Constitución Política de Colombia en su artículo 216, establece que la fuerza pública estará integrada en forma exclusiva por las fuerzas militares y la policía nacional.

La nación tendrá para su defensa unas fuerzas militares permanentes constituidas el por el Ejército, la Armada y la Fuerza Aérea. Las fuerzas militares tendrán como finalidad primordial la defensa de la soberanía, la independencia, la integridad del territorio nacional y del orden constitucional. Art 217.

La policía Nacional es un cuerpo armado permanente de naturaleza civil, a cargo de la nación, cuyo fin primordial es el mantenimiento de las condiciones necesarias para el ejercicio de los derechos y libertades públicas, y para asegurar que los habitantes de Colombia convivan en paz. Constitución política de Colombia art. 218.

\section{Desempeño de la fuerzas militares en el municipio de San Jacinto, Bolívar}

Las fuerzas militares en san Jacinto Bolívar también han realizado muy efectivamente su labor de consolidar la seguridad democrática en la región y sus objetivos están siendo encaminados a cumplir varias metas como son:

- Implementar estrategias para llegar a la comunidad con programas de desarrollo educativo a través de la construcción de aulas escolares que se logran gracias a los recursos de algunas empresas privadas.

- Apoyo a los diferentes planteles educativos, a través de la cátedra de derechos humanos.

- Creación de espacios recreativos como parques de diversión en los barrios La Paz, 8 de Diciembre, el corregimiento el Bongal. Su propósito es acercarse a la comunidad a través de estas obras lo cual ha generado mayor confianza con las fuerzas militares.

- La inteligencia y el patrullaje móvil en todos los corregimientos el cual está conformado por 15 o 20 hombres de infantería de marina con presencia constante en las zonas periféricas y críticas tanto en la zona urbana como rural, con el propósito de controlar la cesación del secuestro, las extorciones, hurto y hechos generadores de perturbación del orden público.

Los resultados han sido extraordinarios, existe una respuesta de la comunidad frente a la institucionalidad, donde existe confianza hacia la infantería de marina, policía nacional que en años anteriores por temor a represalias de los grupos organizados con ajusticiamiento temían a denunciar. Hoy en día Puede decirse que los ciudadanos no tienen temor de acercarse a las fuerzas armadas y a la policía nacional para asistir a eventos convocados por ellos; A demás existe una interacción permanente de la población, cuya finalidad es garantizar los derechos en las instituciones educativas, sectores con extrema pobreza y el fortalecimiento y la proliferación del comercio.

El accionar de la policía en conjunto con la SIJIN, está dando resultados, se han adelantado operativos, allanamientos, capturas, controlando en un alto porcentaje el comercio de sustancias alucinógenas.

A través del fondo de seguridad, la alcaldía municipal suministro dos motocicletas a la policía nacional para apoyar el "Plan Integral De Convivencia y Seguridad Ciudadana San Jacinto Bolívar", teniendo en cuenta el diagnostico violencia intrafamiliar, drogadicción y otros componentes con influencia en posibles hecho perturbadores de la tranquilidad. 
La administración para fortalecer aún más la seguridad y convivencia de la comunidad adelanta acciones con la comunidad vulnerable, con el fin de dar a conocer la problemática y las reacciones de impacto que se generan en la población, acompañados de los planes pedagógicos de desarme y control al uso de sustancias alucinógenas, en los diferentes centros educativos.

\section{Conclusiones}

Es importante conocer que los Montes de María ubicados dentro de la Región Caribe ha sido una de las zonas del país con gran afectación a causa de la violencia a manos de grupos insurgentes pero hoy es otro el ambiente en el que viven sus pobladores para quienes al parecer los cuadros de violencia y desplazamiento vividos en una época quedaron como recuerdos en sus memorias.

La situación de violencia vivida en San Jacinto Bolívar, no solo fue un problema de un municipio y por ende de sus habitantes, sino que trajo consigo repercusiones que afectaron directamente a la población más vulnerable, por lo tanto es deber del Estado, brindar los estándares mínimos de protección como lo señala la Constitución Política de Colombia de 1991, que despliega una carta de derechos institucionales que a su vez llevan consigo los mecanismos necesarios para defenderlos en caso de que sean vulnerados estén o no en una situación de desplazamiento. Al igual que lo establece la reciente ley de justicia y paz 975/2005, la ley 1448 del 2011. El Estado debe ser el garante y protector de los ciudadanos brindándoles una plena seguridad que contribuya a una mejor calidad de vida de toda persona.

A partir de la implementación de la política de seguridad democrática iniciada en el gobierno del ex presidente Álvaro Uribe Vélez, se produjo un fortalecimiento y una consolidación de la seguridad democrática en el municipio de San Jacinto Bolívar, así como cambios en la situación de orden público, evidenciándose una disminución del conflicto armado, el apoyo incondicional brindado por la policía y el ejército nacional y por ende en la reparación de las víctimas.

Todo esto se logró gracias al trabajo conjunto entre la administración local y el gobierno nacional, la fuerza pública y la comunidad. A demás esta investigación sugiere seguir consolidando esta seguridad propiciando ambientes de participación entre las organizaciones de población desplazada la administración pública municipal de San Jacinto, el gobierno nacional , la policía y el ejército, para llevar a cabo una mejor política pública y de seguridad democrática y así atender a los principales afectados. Y evitar cualquier amenaza contra la seguridad de la población.

\section{Resultados}

Los resultados de la presente investigación muestran que es importante que el Estado siga estimulando y apoyando a las víctimas del conflicto para que sean promotores de la seguridad que se logre un buen manejo de la administración pública en esta zona del país, permitiendo el desarrollo y el crecimiento de la riqueza dentro del municipio, ya que a causa del desplazamiento se vieron obligados muchos campesinos a dejar sus fuentes de trabajo.

Otro punto importante es establecer una serie de medidas que permitan corregir las desigualdades existentes de manera que se pueda hacer cada vez más estrecha la brecha que separa la injusticia social que hoy por hoy se hace más notable en el departamento y así de esta forma se pueda reparar adecuadamente a las víctimas.

Seguir con los procesos de fortalecimientos presentados por la alcaldía municipal con la realización de los simulacros en las zonas más afectadas (víctimas y victimarios), en cuanto a los casos de violencia intrafamiliar, enfocados a su sensibilización. A demás del desarrollo del programa "Semillas Padres e Hijos" en el cual se les brinde capacitaciones sobre la prevención del abuso y turismo sexual, maltrato infantil, satanismo, pautas de crianza y educación sexual.

Efectuar difusiones radiales y televisivas, organizando actividades culturales y lúdicas alusivas a la prevención de violencia. Participar de los eventos religiosos y demás que organicen otras instituciones con fines de promoción al respeto por la vida y los derechos humanos.

Desarrollar planes pedagógicos de desarme y control al uso de sustancias alucinógenas, en los diferentes centros educativos con el apoyo de la fuerza pública. 


\section{Referencias}

Acción Social. Oficinas Municipio de San Jacinto Bolívar. Alcaldía Municipal. 2008-2011.

Azate Cardona G (1995). Concentración del poder, violencia, clientelismo y democracia participativa en Colombia. Bogotá. Universidad Pontificia Javeriana.

Colombia responde. (2011). Fortalecimiento Gobernabilidad Democrática Montes de María. Ovejas.

CONPES 3460. (2007) Consejo Nacional de Política Económica y Social. Republica de Colombia. Departamento Nacional de Planeación.

Crissien Borrero, T. J. (2008). Cátedra de buen gobierno. Periodo 205-2008. Universidad de la Costa. Barranquilla, Colombia. Edic. Cuc.

Deepa, N. (2000). La voz de los pobres ¿Hay alguien que nos escuche? Madrid, Barcelona. México: Ediciones Mundi-Prensa. 311.

Gómez Pimienta, L. E. (2005). Colombia: la agonía de las instituciones. El estado derrotado. Colombia: net. educativa.

Méndez de Zubillaga, A. (2012). Derechos de las victimas en la normativa internacional y nacional. El papel de las victimas en el proceso de justicia y paz (septiembre de 2011). Memorias del congreso. Universidad Santo Tomas. Bucaramanga.

Ministerio de Defensa Nacional. (2011). Política integral de seguridad y Defensa para la prosperidad. Bogotá: imprenta nacional de Colombia.

Municipio de San Jacinto.Plan de Desarrollo Municipal 2012-2015. Confianza y seriedad con San Jacinto.

Leal Buitrago, F. (2006) La política de seguridad democrática 2002-2005. Revista Análisis Político: cuatro años del Gobierno Uribe, Balances y Perspectivas. Universidad Nacional de Colombia.

Tierno Galván, E. (2007). Thomas Hobbes. Del ciudadano y Leviatán. 6 ed..Tecnos Madrid. España.

Villa Gómez, J. D., Tejada Bermúdez, C., Sánchez Benítez, N., y Téllez Luque, A, M. (2007). Nombrar lo Innombrable. Reconciliación desde la perspectiva de las víctimas. Programa por la paz CINEP. Impresión panamericana. Bogotá:

Younes Moreno, D. (2009). Derecho Constitucional Colombiano. 10 ed.: Ibáñez. Bogotá Colombia.

Páez Barreto, P.V. (2009). Incidencias de la política de seguridad democrática del gobierno de Álvaro Uribe en las relaciones bilaterales con Venezuela. 2002-2008. Tesis Facultad de Relaciones Internacionales. Universidad Colegio Mayor de Nuestra Señora del Rosario. Bogotá.

República de Colombia. Ley 975 de 2005. Por la cual se dictan disposiciones para la reincorporación de miembros de grupos armados organizados al margen de la ley, que contribuyan de manera efectiva a la consecución de la paz nacional y se dictan otras disposiciones para acuerdos humanitarios.

República de Colombia. Constitución Política de Colombia 1991.

República de Colombia. Ley 1448 de 2011. Por las cual se dictan medidas de atención, asistencia y reparación integral a las víctimas del conflicto armado interno.

Waldmann, P (2007). Guerra civil, terrorismo y anonimia social el caso colombiano en un contexto globalizado. Colombia: Norma. 